\title{
Study on the mechanism of Erzhi Pill in the treatment of breast cancer based on network pharmacology
}

\author{
Fahu Yuan ${ }^{1,2}$, Xinghua Liao ${ }^{1}$, Tongcun Zhang ${ }^{1, *}$ \\ ${ }^{1}$. Institute of Biology and Medicine, College of Life and Health Sciences, Wuhan University of Science and Technology, China \\ ${ }^{2}$ School of Medicine, Jianghan University, China
}

\begin{abstract}
Objective: To explore the possible mechanism of Erzhi Pill in the treatment of breast cancer based on network pharmacology. Methods: Through systematic pharmacology database and analysis platform of Traditional Chinese medicine (TCMSP) and literature review, the candidate active ingredients and corresponding targets of Erzhi Pill were retrieved and collected. The UniProt database and the Human Genome Annotation Database (Genecards) were used to compare the results, and the potential target genes were obtained for the coincidence of Erzhi Pill and breast cancer. Cytoscape was used to construct the "candidate component - action target" network of Erzhi Pill. Generate Style from Statistics tool in String database and Cytoscape software was combined to construct protein interaction network.The Kyoto Encyclopedia of Genes and Genomics (KEGG) pathway enrichment analysis and GO classification enrichment analysis for targets of Erzhi Pill were conducted using the Database for Annotation, Visualization and Integrated Discovery (DAVID ) bioinformation resource Database. Results: A total of 21 candidate active components, including Beta-sitosterol, Quercetin, Luteolin, Demethylwedelolactone, Kaempferol and so on, were screened from Erzhi Pill, corresponding to 158 target genes, including vascular endothelial growth factor (VEGF), amino-terminal kinase (C-Jun), proto-oncogene (C-MYC), matrix metalloproteinase-9 (MMP-9), and mainly involved in TNF- $\alpha$, phosphatidylinositol-3-kinase/protein kinase $\mathrm{B}(\mathrm{PI} 3 \mathrm{~K} / \mathrm{Akt})$, tumor suppressor gene $\mathrm{p} 53$, toll-like receptor family and other signaling pathways. Conclusion: This study predicted the possible mechanism of action of Erzhi Pill in the treatment of breast cancer based on the method of network pharmacology, and provided a direction for further research.
\end{abstract}

\section{Background}

The incidence of breast cancer ranks the first among female malignant tumors, and is one of the most common malignant tumors. In recent years, the incidence of breast cancer tends to increase year by year and reach younger age. In general, the prognosis of most breast cancer patients is better after treatment. A study shows that the 5 -year survival rate of Chinese breast cancer patients is $83.2 \%$, which has gradually turned into a chronic disease[1].

Erzhi Pill is made of Ecliptae Herba and Fructus Ligustri Lucidi formula, has been widely used in the treatment of breast cancer in China[2]. Erzhi pill can enhance efficacy and reduce toxicity, relieve depression and improve quality of life in postoperative chemoradiotherapy for breast cancer patients. However, most studies on Erzhi pills are single aspect in vitro experiments or animal experiments, which have great limitations and it is difficult to obtain the overall network of its mechanism of action in vivo[3].

The chemical components of Erzhi Pill are complex, and the treatment of breast cancer has the advantages of multiple components and multiple targets. However, most of the current studies only focus on the effects of drugs on individuals and individual molecules, and the effective active ingredients and mechanism of action of Erzhi Pill in the treatment of breast cell carcinoma are still unclear. The pharmacology of the two drugs is still in the phase of single gene - single target, there are relatively few studies on their integrity. This study based on the method of network pharmacology, get Erzhi Pill main active ingredient, using molecular functions and pathway enrichment analysis forecast its possible mechanism, and the use of molecular docking technology research core components and the core target of interaction, so as to Erzhi Pill provides the reference for the clinical application of treatment of breast cancer, and can provide a reference for the further research in the future.

\section{Materials and Methods}

\subsection{Acquisition of drug active components and corresponding targets}

Respectively with "Ecliptae Herba" and "Fructus Ligustri Lucidi" as keywords, in Traditional Chinese

\footnotetext{
* Corresponding author: *zhangtongcun@wust.edu.cn
} 
Medicine Systems Pharmacology Database and Analysis Platform (TCMSP, https://tcmspw.com/index.php)[4], searching for its chemical composition. Given that not all components can be used as a drug in traditional Chinese medicine is absorbed by human body, combining with the existing literature and platform recommended, limited in the search results of oral bioavailability (OB) is greater than or equal to $30 \%$, class medicinal filtered (DL) is greater than or equal to 0.18 , get 2 drugs in the chemical composition of composite filter condition information, and with its candidate active ingredients as Erzhi Pill.

\subsection{Prediction of target genes of active components}

The protein names of the candidate targets corresponding to the active components of Erzhi Pill were retrieved through the Uniport database (https://www.uniprot.org), the species was defined as human, and the gene names corresponding to the candidate targets and the corresponding UNIPOTKB were obtained.

\subsection{Acquisition of breast cancer-related target genes}

In the human genome annotation databases (Genecards) (https://www.genecards.org) in breast cancer for the keyword search target genes associated with breast carcinoma in Erzhi Pill main active ingredients of the candidate target genes associated with breast carcinoma, comparing the target gene screening of common target genes, as Erzhi Pill potential target for the treatment of breast carcinoma.

\subsection{Construction and analysis of core target interaction network}

The active components that could not act on the related targets of breast cancer were removed, and then the remaining active components and potential targets for the treatment of breast cancer were introduced into Cytoscape software to construct its component-action target network.

\subsection{Biological processes and pathway analysis}

Both Ecliptae Herba and Fructus Ligustri Lucidi functional target genes were introduced into the DAVID database (http://David.Ncifcrf.gov), a gene list was formed, and the Gene Ontology(GO) classification enrichment analysis and Kyoto Encyclopedia of Genes and Genomes(KEGG) enrichment analysis were conducted on the list. The results were saved to select pathways whose KDR values were less than 0.05 , and the top 20 pathways were selected in ascending order according to KDR values.

\section{Results}

\subsection{Candidate active components and candidate targets of Erzhi Pill}

A total of 119 chemical components of Fructus Ligustri ligustri and 48 chemical components of Ecliptae Herba were collected. With $\mathrm{OB} \geq 30 \%$ and $\mathrm{DL} \geq 0.18$ as the limiting conditions, 21 candidate active components were screened out, among which 13 were belong to Fructus Ligustri ligustri and 10 were belong to Ecliptae Herba. Moreover, 211 target genes of candidate active components of Erzhi Pill were obtained on the platform(Fig.1).

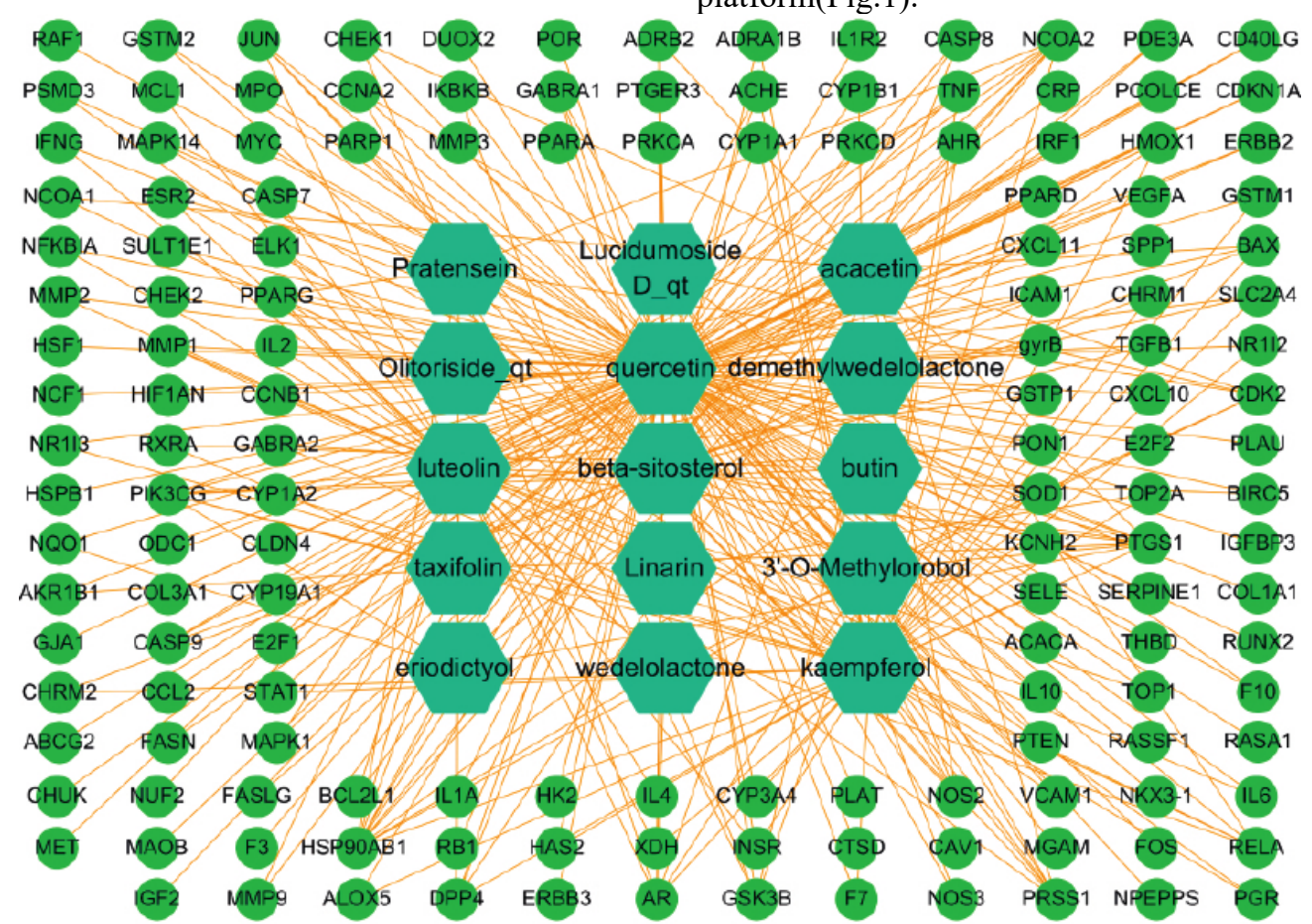

Fig 1. Component-target network of Erzhi Pill 


\subsection{Candidate target gene prediction}

The corresponding genes of 211 candidate targets were obtained by inputing protein targets in Uniprot database. The obtained target genes were compared with the breast cancer-related genes searched in GeneCards database, and 158 potential targets related to breast cancer were screened out.

\subsection{Component-target network construction}

The active components and targets related to breast cancer were introduced into the Cytoscape software, and a component-target network was constructed. The network consisted of 173 nodes, of which 15 nodes were active components, 158 nodes were targets and 338 edges. Different targets can correspond to the same active components, and the same target can also correspond to different active components. It can be seen that Erzhi Pill has the characteristics of multi-component and multitarget in the treatment of breast cancer.

\subsection{Enrichment analysis of GO and KEGG pathways}

The potential targets corresponding to the active components of Erzhi Pill were screened by GO and
KEGG analysis using DAVID database. The pathway with a lower KDR value was more closely related to the treatment of breast cancer with Erzhi Pill. GO enrichment analysis consisted of three parts, namely, cellular component (CC), molecular function (MF) and biological process (BP). The GO analysis results showed that the ones with low KDR value in $\mathrm{CC}$ analysis were the membrane raft, the membrane microdomain,cyclindependent protein kinase holoenzyme complex, Serine /threonine protein kinase complex,transcription factor complex, etc. . MF analysis included nuclear receptor activity, transcription factor activity, doping hormone receptor activity, ubiquitin protein ligase binding, oxidoreductase activity, etc. BP in the analysis for the response to lipopolysaccharide, response to metal ion, the response to reactive oxygen species, the response to nutrient levels, the response to steroid hormone, etc. (Fig.2). KEGG pathway enrichment analysis found that two to pill in the treatment of breast cancer mainly targets with AGE - RAGE signaling Pathway in diabetic activity, Fluid shear stress and atherosclerosis, TNF- $\alpha$ signaling pathway, IL-17 signaling pathway,Apoptosis, PI3K-Akt signaling pathway and other pathways are closely related (Fig.3).

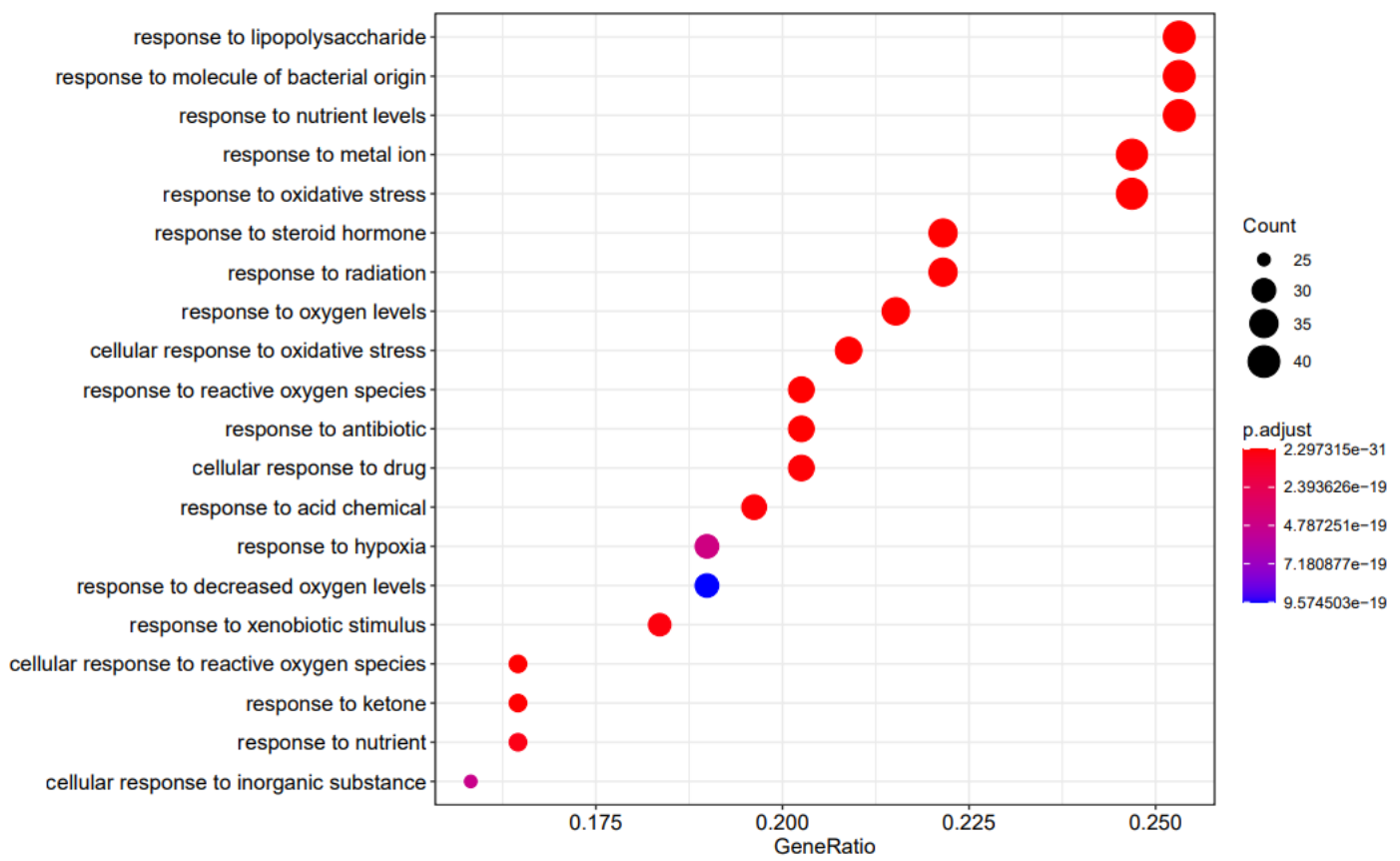

Fig 2. BP analysis on candidate targets from active components of Erzhi Pill 


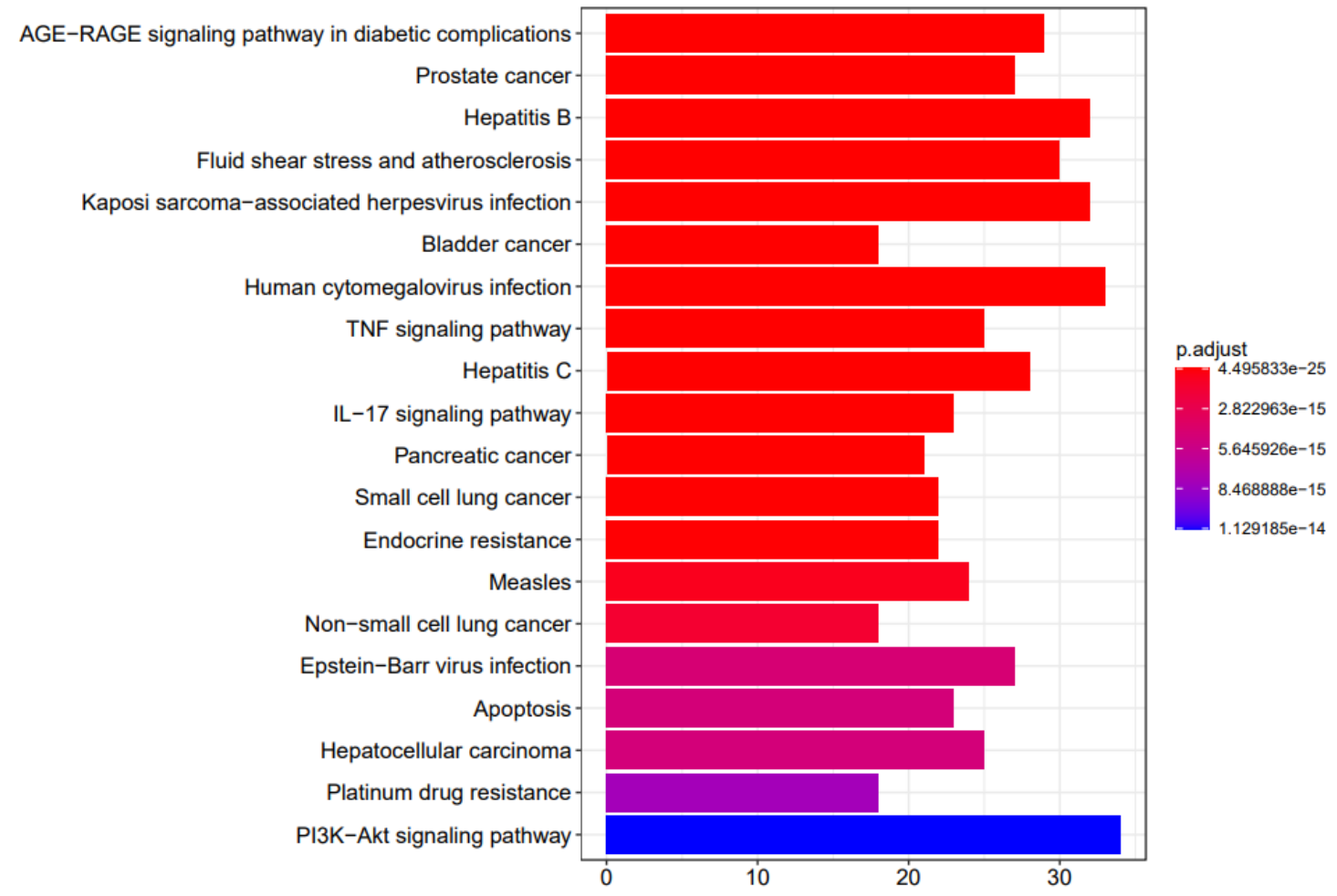

Fig 3. KEGG pathway enrichment analysis of candidate targets from active components of Erzhi Pill

\section{Conclusion}

Ingredients - target network targets in the study of the interaction network embodies the Erzhi Pill more ingredients for the characteristics of target for breast cancer, mechanism of action is complicated in this study showed that Erzhi Pill treating breast cancer are the main active ingredient may be beta-sitosterol, demethylwedelolactone, kaempferol, quercetin, luteolin et $a l$., these active ingredients play a therapeutic role in breast cancer by acting on TNF- $\alpha$,VEGF, C-Jun, C-MYC and MMP-9, and by regulating TNF- $\alpha$, PI3K/Akt, p53, Toll-like receptor and other pathways.

\section{References}

1. Fan L, Strasser-Weippl K, Li J J , et al. Breast cancer in China[J]. Lancet Oncology, 2014, 15(7):e279-e289.

2. Ying W L . Study on Hepatic Protection and Decrease of Enzyme of Erzhi Pill[J]. Strait Pharmaceutical Journal, 2002.

3. Li X, Lu X, Fan D, et al. Synergistic Effects of Erzhi Pill Combined With Methotrexate on Osteoblasts Mediated via the Wnt1/LRP5/ $\beta$-Catenin Signaling Pathway in Collagen-Induced Arthritis Rats[J]. Frontiers in Pharmacology, 2020, 11:228.

4. Ru J, Li P, Wang J, et al. TCMSP: a database of systems pharmacology for drug discovery from herbal medicines[J]. J Cheminform, 2014, 6(1):13. 